\title{
NONLINEAR CONTINUOUS-TIME \\ REFERENCE GOVERNORS FOR CONSTRAINED NONLINEAR SYSTEMS
}

\author{
Hisakazu Nakamura* Nami Kidane* \\ Yuh Yamashita ** Kenichi Kato* \\ Hirokazu Nishitani * \\ * Graduate School of Information Science \\ Nara Instititue of Science and Technology \\ Email: hisaka-n@is.aist-nara.ac.jp,nami-ki@is.naist.jp, \\ kenich-k@is.naist.jp, nisitani@is.aist-nara.ac.jp \\ ** Graduate School of Information Science and Technology \\ Hokkaido University \\ Email: yuhyama@ist.hokudai.ac.jp
}

\begin{abstract}
This paper proposes a reference management technique for closedloop systems with input constraints. A management rule is given by a nonlinear continuous time differential system based on the input-to-state stability and the control Lyapunov approach. Moreover, we confirm the effectiveness of our proposed method by computer simulation. Copyright (C)2005 IFAC
\end{abstract}

Keywords: nonlinear control systems, reference signals, constraints, stability analysis

\section{INTRODUCTION}

Input constraints are ubiquitous characteristics of practical control systems. It is known that violations of constraints drastically degrade system performance, in the worst case leading to instability.

In recent years, many control approaches have been proposed for systems with input constraints. In particular, reference governor control schemes have received considerable attention (Bemporad, 1998),(Gilbert and Kolmanovsky, 2002),(Sugie and Yamamoto, 2001),(Kogiso and Hirata, 2003). However, most reference governors have been considered for discrete-time systems, and all of the reference governors impose a large computation cost.

On the other hand, several control methods have been proposed for nonlinear continuous-time sys- tems with an input constraint using a control Lyapunov approach (Malisoff and Sontag, 2000),(Lin and Sontag, 1995).

In this paper, we propose a reference management technique for nonlinear continuous-time systems with input constraints that have low computation cost. Our proposed method is based on the inputto-state stability and the control Lyapunov approach. Moreover, we confirm the effectiveness of the proposed method by computer simulation.

\section{PRELIMINARIES}

In this section, we briefly explain important definitions and properties (Isidori, 1999),(Sontag and Wang, 1995). 


\subsection{Control Lyapunov function and stability}

In this subsection, we consider the following inputaffine system.

$$
\dot{x}=f(x)+g(x) \cdot u,
$$

where $x \in \mathbb{R}^{n}$ is state, $u \in U \subseteq \mathbb{R}^{m}$ is input, $f: \mathbb{R}^{n} \rightarrow \mathbb{R}^{n}, g: \mathbb{R}^{m} \rightarrow \mathbb{R}^{n}$ are continuous mapping, and $f(0)=0$.

Definition 1. (Control Lyapunov function). A radially unbounded function $V: \mathbb{R}^{n} \rightarrow \mathbb{R}_{+}$is a control Lyapunov function if the following inequality is satisfied:

$$
\inf _{u \in U}\left\{L_{f} V+L_{g} V \cdot u\right\}, \quad \forall x \neq 0 .
$$

Definition 2. (Local control Lyapunov function). A smooth radially-unbounded positive-definite function $V$ is said to be a locally control Lyapunov function if the following inequality is satisfied:

$$
\inf _{u \in U}\left\{L_{f} V+L_{g} V \cdot u\right\}<0, \quad \forall x \neq 0,
$$

where state $x$ is in the neighborhood of the origin.

\subsection{Input-to-state-stability}

We consider the following system in this subsection:

$$
\dot{x}=f(x, u),
$$

where $f: \mathbb{R}^{n} \times \mathbb{R}^{m} \rightarrow \mathbb{R}^{n}$ satisfies $f(0,0)=0$.

Definition 3. (Input-to-state-stability (ISS)).

System (4) is said to be input-to-state stable if there exist a class KL function $\beta(\cdot, \cdot)$ and a class $K$ function $\gamma(\cdot)$, called a gain function, such that for any input $u(\cdot) \in L_{\infty}^{m}$ and any $x_{0} \in \mathbb{R}^{n}$, the response of $x(t)$ of system (4) in the initial state $x(0)=x_{0}$ satisfies

$$
\|x(t)\| \leq \beta\left(\left\|x_{0}\right\|, t\right)+\gamma\left(\|u(\cdot)\|_{\infty}\right)
$$

for all $t \geq 0$.

Definition 4. (ISS-Lyapunov function). A smooth function $V: \mathbb{R}^{n} \rightarrow \mathbb{R}_{+}$is called an ISS-Lyapunov function for system (4) if there exist $K_{\infty}$-functions $\underline{\alpha}_{1}, \bar{\alpha}_{1}$, and $K$ functions $\alpha_{2}$ and $\xi$, such that

$$
\underline{\alpha}_{1}(\|x\|) \leq V(x) \leq \bar{\alpha}_{1}(\|x\|)
$$

for any $x \in \mathbb{R}^{n}$ and

$$
\frac{\partial V}{\partial x} f(x, u) \leq-\alpha_{2}(\|x\|)
$$

for any $x \in \mathbb{R}^{n}$ and any $u \in \mathbb{R}^{m}$ so that $\|x\| \geq$ $\xi(\|u\|)$.

Lemma 1. A smooth function $V$ is an ISS-Lyapunov function for system (4) if and only if there exist $K_{\infty}$ functions $\underline{\alpha}_{1}, \bar{\alpha}_{1}, \alpha_{2}, \alpha_{3}$ such that eq. (6) holds, and

$$
\frac{\partial V}{\partial x} f(x, u) \leq-\alpha_{2}(\|x\|)+\alpha_{3}(\|u\|) .
$$

Theorem 1. The system is ISS if and only if it admits an ISS-Lyapunov function.

Moreover, we use the following corollary shown in (Isidori, 1999) in the following section.

Corollary 1. Consider the following cascade connected nonlinear system:

$$
\begin{aligned}
& \dot{x}=f(x, z) \\
& \dot{z}=g(z),
\end{aligned}
$$

where $x \in \mathbb{R}^{n}, z \in \mathbb{R}^{m}, f(0,0)=0, g(0)=$ 0 , and $f(x, z), g(z)$ are continuous. Suppose the following subsystem $\dot{x}=f(x, z)$ viewed as a system with input $z$ and state $x$ is input-tostate stable and that the equilibrium $z=0$ of $\dot{z}=g(z)$ is globally asymptotically stable. Then, the equilibrium $(x, z)=(0,0)$ of system $(9)$ is globally asymptotically stable.

\subsection{Stabilization with an input constraint}

In this subsection, consider the following singleinput single-output (SISO) system:

$$
\dot{x}=f(x)+g(x) \cdot u,
$$

where $x \in \mathbb{R}^{n}$ is state and $u \in U \subseteq \mathbb{R}^{m}$ is input. We assume $f: \mathbb{R}^{n} \rightarrow \mathbb{R}^{n}, g: \mathbb{R}^{n} \rightarrow \mathbb{R}^{n \times m}$ is smooth and $f(0)=0$.

For system (10), the following theorem has been proposed in (Lin and Sontag, 1995):

Theorem 2. Let a control Lyapunov function $V(x)$ be known for system (10). Then, the input

$$
u= \begin{cases}-\frac{L_{f} V+\sqrt{\left(L_{f} V\right)^{2}+\left(L_{g} V\right)^{4}}}{L_{g} V\left(1+\sqrt{\left.1+\left(L_{g} V\right)^{2}\right)}\right.} & \left(L_{g} V \neq 0\right) \\ 0 & \left(L_{g} V=0\right)\end{cases}
$$

stabilizes the system and satisfies the following input constraint:

$$
\|u\|<1
$$

We use the following corollary in the following sections:

Corollary 2. Let a control Lyapunov function $V(x)$ be known for system (10). Then, the input 


$$
u=\left\{\begin{array}{cc}
-\frac{L_{f} V+\sqrt{\left.\left(L_{f} V\right)^{2}+C(\|x\|) \cdot L_{g} V\right)^{4}}}{L_{g} V\left(1+\sqrt{\left.1+\left(C(\|x\|) \cdot L_{g} V\right)^{2}\right)}\right.} \\
0 & \left(L_{g} V \neq 0\right) \\
& \left(L_{g} V=0\right)
\end{array}\right.
$$

stabilizes the system and satisfies the following input constraint:

$$
\|u\|<C(\|x\|) \quad C(\|x\|)>0)
$$

Proof is easy and omitted.

\section{NONLINEAR REFERENCE GOVERNORS}

In this paper, we construct a continuous-time reference governor for a nonlinear system. First, we construct the 'nominal' reference governor, and then we construct a 'robust' reference governor.

We consider the following SISO system in this paper:

$$
\begin{aligned}
\dot{x} & =f(x, u) \\
y & =c(x) \\
u & =k(x, r)
\end{aligned}
$$

where $x \in \mathbb{R}^{n}$ is state, $y \in \mathbb{R}^{1}$ is an output of the system, $u \in \mathbb{R}^{1}$ is an input, and $r \in \mathbb{R}^{1}$ is a reference signal. Moreover, we suppose the following initial condition:

$$
x(0)=r(0)=0 .
$$

In this system, input $u$ has the following input constraint.

$$
u \in U \subset \mathbb{R}^{1},
$$

where

$$
0 \in U \text {. }
$$

Hence, if residual $r-y$ is large, the input becomes large and the constraint (17) may not be satisfied.

The problem of this paper is to construct a management system of a reference signal $r$ that $u$ satisfies the input constraint for any desired output $w$.

In this paper, we suppose the following additional assumptions:

Assumption 1. When there does not exist an input constraint, we assume system (15) is asymptotically stable when $r$ is a constant. Moreover, we can describe the controlled system by the following error system:

$$
\dot{\tilde{x}}=\tilde{f}(\tilde{x}),
$$

where $\tilde{x}=0$ when $c(x)=r$.
Assumption 2. When $r$ is time-varying, we assume eq. (19) can be described by the following equation.

$$
\dot{\tilde{x}}=\tilde{f}(\tilde{x})+g(\tilde{f}) \cdot \dot{r} .
$$

Assumption 3. System (20) is ISS for input $\dot{r}$ and state $\tilde{x}$.

Under assumption 3, there exist the following ISS Lyapunov functions by lemma 1 :

$$
\begin{aligned}
\underline{\alpha}_{1}(\|x\|) & \leq V(\tilde{x}) \leq \bar{\alpha}_{1}(\|x\|) \\
\dot{V}(\tilde{x}) & \leq-\alpha_{2}(\|\tilde{x}\|)+\alpha_{3}(\|\dot{r}\|) .
\end{aligned}
$$

Then, we suppose the following assumption:

Assumption 4. An ISS Lyapunov function $V$ and function $a(\|x\|)$ is known such that when $V \leq$ $a(\|x\|), u \in U$.

Assumption 5. When $r(0)=y(0), V(\tilde{x}(0))<$ $a(\|x\|)$.

Assumption 6. The desired output $w$ is constant, namely,

$$
w(t) \equiv w .
$$

Based on these hypotheses, the problem of this paper is to construct the following control system, $\mathrm{m}$ in which $r$ converges to $w$ when $V$ is small and decreases $V$ when the value of $V$ is close to $a(\|x\|)$ :

$$
\begin{aligned}
\dot{v} & =\hat{f}(v, w, V(\tilde{x})) \\
r & =\hat{c}(v) .
\end{aligned}
$$

We call system (24) a reference governor system.

\subsection{Construction of a nominal reference governor}

When $\dot{r}=0$ and $V \leq a, \tilde{x}$ is asymptotically stable and the input constraint is satisfied by eq. (19),eq. (20) and assumption 4.

In this subsection, we show the sufficient condition of $\dot{V}<0$ when $V=a$ by the property of ISS.

The following equation is held by the property of the ISS Lyapunov function $V$ :

$$
\dot{V} \leq-\alpha_{2}\left(\underline{\alpha}_{1}^{-1}(V(\tilde{x}))\right)+\beta(|\dot{r}|) .
$$

Therefore, if

$$
\alpha_{2}\left(\underline{\alpha}_{1}^{-1}(a(\| x||)) \geq \beta(|\dot{r}|),\right.
$$

$\dot{V}<0$ when $V(\tilde{x})=a(\|x\|)$. Then, $V$ is decreasing and input constraint is satisfied. By eq. (26), if $\dot{r}$ satisfied the following equation;

$$
|\dot{r}| \leq c(\|x\|)=\beta^{-1} \circ \alpha_{2} \circ \underline{\alpha}_{1}^{-1}(a(\|x\|)),
$$


the input $u$ satisfies the input constraint. Hence, we consider the following the single-order reference governor system:

$$
\begin{aligned}
\dot{\hat{x}} & =v \\
r & =\hat{x},
\end{aligned}
$$

where $\hat{x}$ and $v \in \mathbb{R}^{1}$. Then, the problem of constructing a reference governor for system (15) becomes that of constructing the controller $v$ that the following system is asymptotically stable when $r$ is constant:

$$
\frac{d}{d t}(\hat{x}-w)=v(\hat{x}, w),
$$

where $v$ satisfies the following input constraint:

$$
|v| \leq C(\|x\|)
$$

We consider the following Lyapunov function candidate $W(\tilde{x})$ :

$$
W(\hat{x}-w)=\frac{1}{2} k_{3}(\hat{x}-w)^{2} .
$$

Then, if we choose the input $v$ as

$$
\begin{aligned}
v & =-\frac{\left(C(\|x\|) \cdot L_{g} W\right)^{2}}{L_{g} V\left(1+\sqrt{1+\left(C(\|x\|) \cdot L_{g} W\right)^{2}}\right)} \\
& =-\frac{C(\|x\|)^{2} k_{3}(\hat{x}-w)}{1+\sqrt{1+C(\|x\|)^{2} k_{3}^{2}(\hat{x}-w)^{2}}},
\end{aligned}
$$

system (30) is asymptotically stable and satisfies the input constraint (17).

Therefore we have the following theorem.

Theorem 3. Consider system (15) and suppose assumption $1 \sim 6$. Then, the following reference governor stabilize the system and the input $u$ satisfies the input constraint $u \in U$.

$$
\begin{aligned}
\dot{\hat{x}} & =-\frac{C(\|x\|)^{2} k_{3}(\hat{x}-w)}{1+\sqrt{1+C(\|x\|)^{2} k_{3}^{2}(\hat{x}-w)^{2}}} \\
r & =\hat{x} \\
\hat{x}(0) & =0 .
\end{aligned}
$$

Proof. Consider the cascade connected system system (15) and system (50). Because system (15) is ISS and system (50) is asymptotically stable, it is clear that the connected system is asymptotically stable by corollary 1 . Moreover, $\|\dot{r}\|<C(\|x\|)$, the input constraint is always satisfied.

\subsection{Construction of robust reference governor}

The reference governor proposed in the previous subsection always decrease the error $r-w$ independent of the value of $V(\tilde{x})$. Therefore, if some disturbances are added to the system, the values of $V(\tilde{x})$ may become larger than $a$. Then, we consider the following system with the disturbance $p(t)$ which satisfies the matching condition:

$$
\dot{\tilde{x}}=\tilde{f}(\tilde{x})+g(\tilde{x}) \cdot(\dot{r}+p(t)),
$$

where, $p(t)$ is a bounded function. The problem in this subsection is to construct the following reference governor system that decrease the value of $V(\tilde{x})$ when $V(\tilde{x})$ is larger than an arbitrary constant $\tilde{C}(\|x\|)<C(\|x\|)$ :

$$
\begin{aligned}
& \dot{\hat{x}}=v \\
& r=\hat{x} .
\end{aligned}
$$

In the previous subsection, we have designed the following nominal reference governor. Hence, the reference governor that satisfies $|\dot{r}| \leq \tilde{C}(\|x\|)$ can be obtained as the following system:

$$
\begin{aligned}
\dot{\hat{x}} & =-\frac{\tilde{c}^{2} k_{3}(\hat{x}-w)}{1+\sqrt{1+\tilde{C}(\|x\|)^{2} k_{3}^{2}(\hat{x}-w)^{2}}} \\
r & =\hat{x} \\
\hat{x}(0) & =0 .
\end{aligned}
$$

Then, we define $\tilde{a}>0$ as

$$
\tilde{a}(\|x\|)=\underline{\alpha}_{1} \circ \alpha_{2}^{-1} \circ \beta(\tilde{C}(\|x\|)) .
$$

For preserving the input constraint (17), we decrease the value of $V(\tilde{x})$ when

$$
\tilde{a}<V(\tilde{x}) \leq a(\|x\|) .
$$

We consider the ISS Lyapunov function $V(\tilde{x})$ for system (20). Then, the derivative of $V$ can be described by the following equation:

$$
\dot{V}=L_{\tilde{f}} V+L_{g} V \cdot(\dot{r}+p(t)) .
$$

We construct the $\dot{r}$ as the following equation:

$$
\dot{r}=-\frac{a L_{g} V}{a-V(\tilde{x})} \eta(V(\tilde{x}) \mid),
$$

where the function $\eta$ is defined by the following equations:

$$
\begin{aligned}
\eta(x) & =0 \\
\eta(x) & =\left(\frac{x-\tilde{a}(\|x\|)}{a(\|x\|)-\tilde{a}(\|x\|)}\right)^{2}(x \leq \tilde{a}(\|x\|)) \\
& (\tilde{a}(\|x\|)<x<a(\|x\|)) .
\end{aligned}
$$

Then,

$$
\dot{V}<L_{g} V \cdot\left\{-\frac{a L_{g} V}{a(\|x\|)-V(\tilde{x})} \eta(V(\tilde{x}))+p(t)\right\}
$$

$$
<0 \text {. }
$$

Then, we can obtain the following lemma.

Lemma 2. Consider system (15) and suppose assumption $1 \sim 6$. Then, when we choose $\dot{r}$ as eq. (45), $V(\tilde{x}) \leq a$ always holds. 
Proof . To prove the lemma, we prove there exists $\bar{a} \leq a(\|x\|)$ such that $\dot{V}(x)<0$ for all $x \in$ $\{x \mid\|x\|=\bar{a}\}$.

(1) Case: $L_{g} V \neq 0$

By assumption, there exists $\bar{p}$ such that $\|p(t)\| \leq \bar{p}$. Then,

$$
\dot{V}<L_{g} V \cdot\left\{-\frac{a L_{g} V}{a-V(\tilde{x})} \eta(V(\tilde{x}))+\bar{p}\right\} .
$$

Then, there exists $V(x)<a$ such that

$$
\frac{a}{(a-V(\tilde{x}))} \eta(V(\tilde{x}))>\bar{w} .
$$

Therefore, $V(x)<a$ holds.

(2) Case $L_{g} V=0$

By

$$
\dot{V}=L_{f} V<0,
$$

$$
V(x)<a \text { holds. }
$$

Therefore when we choose $\dot{r}$ as eq. (45), $V(\tilde{x}) \leq a$ always holds.

Therefore, note that $\dot{\hat{x}}=\dot{\dot{r}}$, we can construct the following robust reference governor:

$$
\begin{aligned}
\dot{\hat{x}}= & -\frac{C(\|x\|)^{2} k_{3}(\hat{x}-w)}{1+\sqrt{1+C(\|x\|)^{2} k_{3}^{2}(\hat{x}-w)^{2}}} \\
& -\frac{a L_{g} V}{a(\|x\|)-V(\tilde{x})} \eta(V(\tilde{x})) \\
r= & \hat{x} \\
\hat{x}(0)= & 0 .
\end{aligned}
$$

Finally, we can obtain the following theorem:

Theorem 4. Consider system (15) and suppose assumption $1 \sim 3$. Then, the following reference governor holds $V(\tilde{x}) \leq a$, and if $p(t)=0$, the output of system (15) converges to the origin.

$$
\begin{aligned}
\dot{\hat{x}}= & -\frac{C(\|x\|)^{2} k_{3}(\hat{x}-w)}{1+\sqrt{1+C(\|x\|)^{2} k_{3}^{2}(\hat{x}-w)^{2}}} \\
& -\frac{a(\|x\|) L_{g} V}{a(\|x\|)-V(\tilde{x})} \eta(V(\tilde{x})) \\
r= & \hat{x} \\
\hat{x}(0)= & 0 .
\end{aligned}
$$

\section{EXAMPLE}

In this section, we construct the reference governor for the following system:

$$
\begin{aligned}
\dot{x} & =u \\
y & =x \\
u & =-(x-r),
\end{aligned}
$$

where $x, y, u \in \mathbb{R}^{1}$ and the input $u$ has the following input constraint.

$$
|u| \leq 1
$$

The error system of system (51) can be described by the following equations:

$$
\dot{\tilde{x}}=-\tilde{x}+\dot{r},
$$

where $\tilde{x}=x-r$. Then, consider the following function $V$ :

$$
V(\tilde{x})=\frac{1}{2} \tilde{x}^{2} .
$$

Then, the derivative of $V$ can be described by the following equation:

$$
\begin{aligned}
\dot{V} & =-\tilde{x}^{2}+\tilde{x} \dot{r} \\
& \leq-\frac{1}{2} \tilde{x}^{2}+\frac{1}{2} r^{2} .
\end{aligned}
$$

Therefore $V$ is an ISS Lyapunov function.

The input constraint $|u| \leq 1$ coincides $V \leq 1$ because of eq. (51) and eq. (55). Therefore we can obtain

$$
c=1 \text {. }
$$

Consider the following Lyapunov function candidate $W(\tilde{x})$

$$
W(\hat{x}-w)=5(\hat{x}-w)^{2}
$$

We can construct the following nominal reference governor:

$$
\begin{aligned}
\dot{\hat{x}} & =-\frac{10(\hat{x}-w)}{1+\sqrt{1+100(\hat{x}-w)^{2}}} \\
r & =\hat{x} \\
\hat{x}(0) & =0 .
\end{aligned}
$$

Next, we construct the robust reference governor for the following system with disturbance:

$$
\begin{aligned}
& \dot{x}=u+p(t) \\
& y=x \\
& u=-(x-r),
\end{aligned}
$$

Then, the derivative of $V$ defined in eq. (54) is shown in the following equation:

$$
\dot{V}=-\tilde{x}^{2}+\tilde{x}(\dot{r}+p(t)) .
$$

Therefore, disturbance $p(t)$ satisfies the matching condition.

We choose $\tilde{c}=0.9$. Then, $\tilde{a}=0.5 \cdot 0.9^{2}$. Therefore we can construct the following robust reference governor: We can construct the following nominal reference governor:

$$
\begin{aligned}
\dot{\hat{x}}= & \frac{10 \cdot 0.9^{2}(\hat{x}-w)}{1+\sqrt{1+100 \cdot 0.9^{2}(\hat{x}-w)^{2}}} \\
& +\frac{x-r}{0.5-0.5(x-r)^{2}} \eta\left(\frac{1}{2}(x-r)^{2}\right) \\
r= & \hat{x} \\
\hat{x}(0)= & 0,
\end{aligned}
$$

where

$$
\begin{array}{ll}
\eta(x) & =0 \\
\eta(x) & =\left(\frac{x-0.5 \cdot 0.9^{2}}{0.5-0.5 \cdot 0.9^{2}}\right)^{2} \quad\left(0.5 \cdot 0.9^{2}<x<0.5\right) .
\end{array}
$$


We show the results of the computer simulation with the desired output $w=5$. The results of the nominal reference governor is shown in the Fig. 1 and 2. By Fig. 1, we can confirm the state $x$ converges to the desired output $w=5$. By Fig. 2, the input does not exceed the input constraint 1.

In the Fig. 3 and 4, we show the simulation results of the robust reference governor with Gaussian distribution disturbance that standard deviation is equal to 500. By Fig. 3, we can admit the disturbance is very large. However, the input preserves their constraint by Fig. 4 .

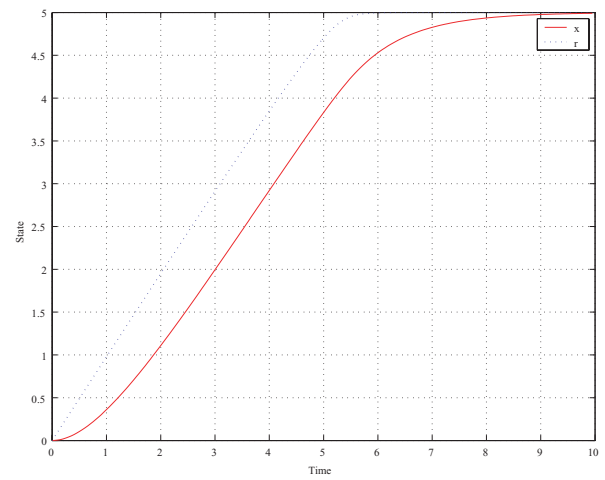

Fig. 1. Simulation Results - Nominal Reference Governor: State

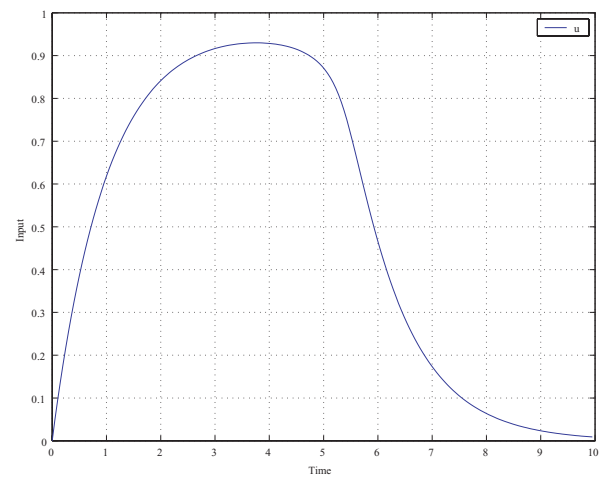

Fig. 2. Simulation Results - Nominal Reference Governor: Controller Output

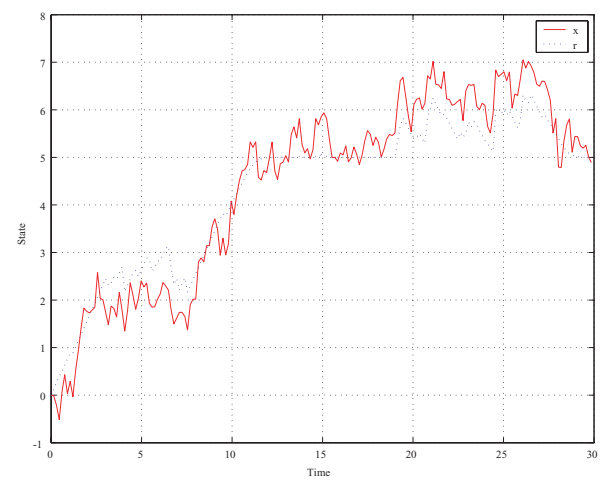

Fig. 3. Simulation Results - Robust Reference Governor: State

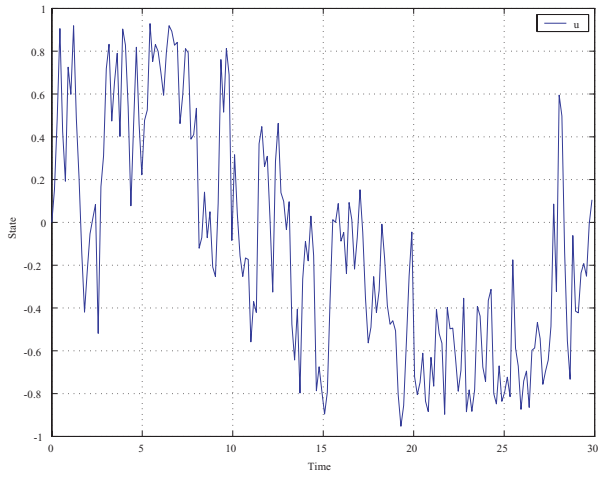

Fig. 4. Simulation Results - Robust Reference Governor: Controller Output

\section{CONCLUSION}

In this paper we have proposed two reference governors for nonlinear systems with input constraints. The reference governors proposed in this paper is continuous-time systems, and constructed based on the input-to-state stability and control Lyapunov function. Moreover, we have confirmed the effectiveness of our proposed method by computer simulations.

\section{REFERENCES}

Bemporad, A. (1998). Reference governor for constrained nonlinear systems. IEEE Transactions on Automatic Control.

Gilbert, E. and I. Kolmanovsky (2002). Nonlinear tracking control in the presence of state and control constraints: a generalized reference governor. Automatica 38, 2063-2073.

Isidori, A. (1999). Nonlinear Control Systems II. Springer. London.

Kogiso, K. and K. Hirata (2003). A reference governor in a piecewise state affine function. Proceedings of the 42nd IEEE Conference on Decision and Control pp. 1747-1752.

Lin, Y. and E. D. Sontag (1995). ControlLyapunov universal formulas for restricted inputs. Control-Theory and Advanced Technology 10, 1981-2004.

Malisoff, M. and E. D. Sontag (2000). Universal formulas for feedback stabilization with respect to Minkowski balls. Systems \& Control Letters 40, 247-260.

Sontag, E. D. and Y. Wang (1995). On characterizations of the input-to-state stability property. Systems \& Control Letters 24, 351-359.

Sugie, T. and H. Yamamoto (2001). Reference management for closed loop systmes with state and control constraints. Proceedings of the American Control Conference 2001 pp. 1426-1431. 